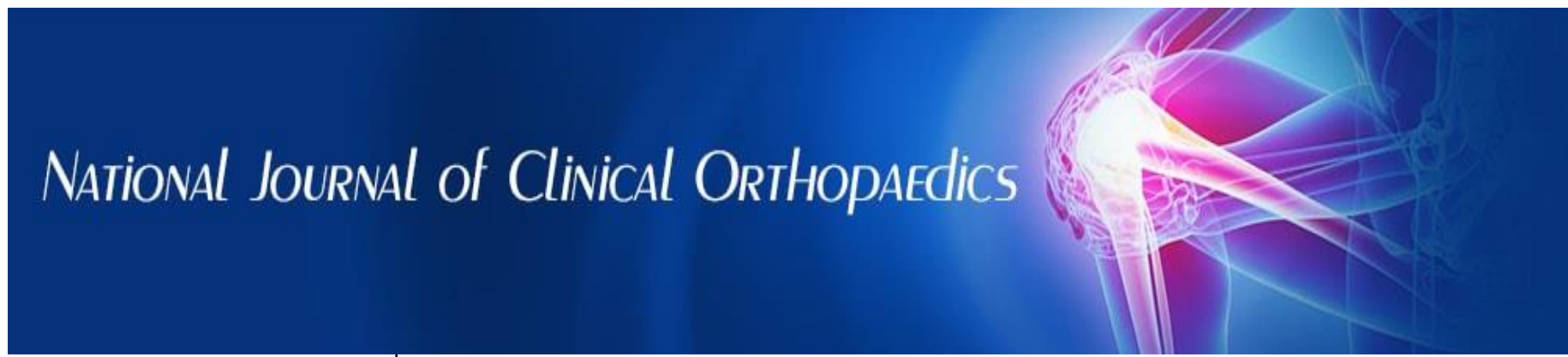

ISSN (P): 2521-3466

ISSN (E): 2521-3474

(C) Clinical Orthopaedics

www.orthoresearchjournal.com

2020; 4(1): 29-32

Received: 16-11-2019

Accepted: 20-12-2019

\section{Karim-Ullah Khan}

Resident in the Department of Orthopedics in UCMS and GTB Hospital, Delhi, India

\section{Apoorv Sehgal}

Senior Resident in the

Department of Orthopedics in UCMS and GTB Hospital, Delhi, India

\section{Pratyush Shah}

Resident in the Department of Orthopedics in UCMS and GTB Hospital, Delhi, India

\section{Deepak Singh}

Senior Resident in the

Department of Orthopedics in UCMS and GTB Hospital, Delhi, India

\section{Sushil Kamal}

Senior Resident in the

Department of Orthopedics in UCMS and GTB Hospital, Delhi, India
Corresponding Author: Apoorv Sehgal Senior Resident in the Department of Orthopedics in UCMS and GTB Hospital, Delhi, India

\section{Outcome of delayed surgery in gartland type III supracondylar fractures of humerus in children}

\author{
Karim-Ullah Khan, Apoorv Sehgal, Pratyush Shahi, Deepak Singh and \\ Sushil Kamal
}

DOI: https://doi.org/10.33545/orthor.2020.v4.i1a.197

\section{Abstract}

Background: Supracondylar fracture of humerus in children constitutes more than half of paediatric elbow injuries requiring hospitalisation. Data over outcomes of delayed surgery in Gartland type III fracture supracondylar humerus in children is scarce and no clear guildelines exist regarding management of such injuries. Hence, we envisaged to study the functional and radiological outcome and complications of delayed surgery (between 2-12 days) in paediatric patients with Gartland type III supracondylar fracture of humerus.

Material and Methods: A prospective interventional study was conducted in 20 patients aged 4-12 years presenting in Orthopaedic OPD / emergency with Gartland type III fracture supracondylar humerus without associated neurovascular compromise and without other injuries who underwent delayed open reduction and cross $\mathrm{K}$ wire fixation through medio-lateral approach. At latest follow up (min 3 months), functional outcome was assessed using Flynn's criteria which includes loss in range of motion (ROM) and loss of carrying angle. Radiological outcomes were assessed using Baumann's angle and anterior humeral line. Complications, if any will be noted.

Results: Mean age was $7.75 \pm 2.38$ years ( $4-12$ years) and mean delay in surgery was of $3.9 \pm 1.65$ days (2-9 days). Out of 20 patients, right sided extremity was involved in six $(20 \%)$ patients while $14(70 \%)$ patients suffered injury over left side. The minimum follow up period was of three months with a mean follow up of $5.54 \pm 0.72$ months. The mean loss in ROM was $21.2^{\circ} \pm 13.5^{\circ}\left(5^{\circ}-55^{\circ}\right)$ at three on this and $12.4^{\circ} \pm 8.8^{\circ}\left(0^{\circ}-30^{\circ}\right)$ at six months. The difference was found to be statistically significant ( $\mathrm{p}$-value $\left.<0.05\right)$. The mean loss of carrying angle was $8.1^{\circ} \pm 6.21^{\circ}\left(1^{\circ}-22^{\circ}\right)$ at three months and was $4.58^{\circ} \pm 5.65^{\circ}\left(0^{\circ}-20^{\circ}\right)$ at six months of follow up which was found to be statistically significant (p-value $<0.005$ ). As per Flynn's criteria, functional outcome was found to be satisfactory in seven $(35 \%)$ patients and unsatisfactory in 13 $(65 \%)$ patients at three months follow up. Twelve patients reached six months follow up period, seven (58.3\%) patients had satisfactory outcome while five (41.7\%) patients had unsatisfactory outcome. Difference in Baumann's angle was found to be statistically insignificant when compared with immediate postop and latest follow up (min three months). During immediate postop anterior humeral line passed through capitellum in $90 \%$ patients and anterior to capitellum in $10 \%$ cases. At latest follow up, it passed through capitellum in $85 \%$ cases and anterior to capitellum in $15 \%$ cases. Postop serous blisters and pin site infection each was found in one $(5 \%)$ patient while cubitus varus was found in four (20\%) patients.

Conclusion: At three months follow up, we achieved satisfactory results in some patients but with continued physiotherapy we were able to achieve satisfactory results in significant proportion of patients at six months follow up. So open reduction with $\mathrm{K}$ wire fixation is a reasonably fair method for tackling delayed presenting Gartland type III fractures supracondylar humerus in children.

Keywords: delayed surgery, gartland type, humerus, children

\section{Introduction}

Supracondylar fracture of humerus is one of the most common fractures in children constituting about $3 \%$ of all fractures ${ }^{[1]}$. These fractures are divided into extension $(98 \%)$ and flexion type $(2 \%)^{[2]}$. Gartland originally described classification system for extension type of supracondylar humerus fractures ${ }^{[3,4]}$.

Displaced supracondylar fractures are conventionally treated with closed reduction and percutaneous pinning on emergency basis ${ }^{[5]}$. However, late presentation and delayed surgery are common in developing countries due to various reasons ${ }^{[6]}$. The reasons of delay could be due to lack of awareness, initial treatment by osteopaths or trauma in areas remote from health 
facilities.

Sometimes primary centres tend to shift patients with excessive swelling and associated neurovascular complications to higher centres leading to further delay in definitive treatment. Operations may be further delayed due to non-availability of operation theatre and shortage of manpower/equipment ${ }^{[7]}$. Late presentation is defined as fractures presenting 2 days after injury $[8,9]$.

Only few reports are available on the treatment with delay of more than 2 days ${ }^{[6,8,9]}$. There remains a dilemma while managing late cases, whether to treat them as fresh supracondylar fracture or to allow them to malunite and consider for corrective surgery at a later stage ${ }^{[10]}$. Few authors have reported the results of delayed open reduction and internal fixation in these fractures from 2-14 days post injury [6, 8]. Unfortunately, in literature there are no clear guidelines or consensus regarding management of delayed presentation of supracondylar fracture ${ }^{[10]}$.

Hence, we envisage to study the functional and radiological outcome and complications of delayed surgery (i.e. after 2-12 days) in paediatric patients with Gartland type III supracondylar fracture of humerus.

\section{Materials and Methods}

This prospective interventional study was conducted at UCMS \& GTB Hospital, Delhi from October, 2017 to April, 2019. 20 patients in the age group of 4 to 12 years with closed Gartland type III supracondylar humerus fracture presenting 48 hours to 12 days after injury were included in the study.

Detailed history was taken regarding date of injury, mechanism of injury, interval between time of injury and presentation, interval between time of presentation and surgery, previous attempt at closed reduction and any intervention from osteopath. After thorough general physical examination, involved extremity was examined for tenderness, swelling and deformity around the elbow. Distal pulses, capillary filling, sensory examination and motor examination were also assessed.

Patients were subjected to posterior above elbow splintage (above elbow slab) and subjected to elbow radiographs (anteroposterior and lateral views). Subsequently, due to unavailability of C-ARM in emergency OT at our institute, patients were posted for open reduction and internal fixation as soon as possible.

Surgery was done in supine position under general anaesthesia. Fracture site was approached using lateral Kocher's approach to the distal humerus. After fracture reduction, fracture was fixed using two crossed percutaneous K-wires (one lateral and one medial). For placement of medial wire a $1-2 \mathrm{~cm}$ incision was given over the medial epicondyle to prevent ulnar nerve injury during wire placement. In case the fracture reduction was difficult from the lateral approach, the surgeon added a formal medial approach to further expose the fracture site to enable satisfactory fracture reduction.

Postoperatively, the elbow was immobilised in an above-elbow POP slab in $90^{\circ}$ elbow flexion. Check x-ray was done after 24 hours, fracture reduction was assessed using Baumann's angle and anterior humeral line. Wound inspection and drain removal was done after 48 hours of surgery.

Subsequently patients were followed up at 1 week, 2 weeks, 4 weeks, 6 weeks, 3 months and 6 months. Functional and radiological assessment was done at a minimum follow up of 3 months or latest follow-up using Flynn's criteria (table 1), Baumann's angle and anterior humeral line ${ }^{[11-14]}$.
Table 1: Flynn's criteria

\begin{tabular}{|c|c|c|c|}
\hline Results & Rating & Loss of carrying angle & Loss of motion \\
\hline \multirow{3}{*}{ Satisfactory } & Excellent & $0-5^{\circ}$ & $0-5^{\circ}$ \\
\cline { 2 - 4 } & Good & $5-10^{\circ}$ & $5-10^{\circ}$ \\
\cline { 2 - 4 } & Fair & $10-15^{\circ}$ & $10-15^{\circ}$ \\
\hline Unsatisfactory & Poor & $>15^{\circ}$ & $>15^{\circ}$ \\
\hline
\end{tabular}

\section{Results}

The average age of patients in our study was 7.8 years with $80 \%$ patients being males. Left elbow was more commonly affected. Fall was the most common mode of injury. The average delay in surgery was 3.9 days with $90 \%$ of the patients being operated within 2-6 days following injury. Patients receiving primary treatment in form of splintage followed by referral to our centre was the most common cause of delay (65\%). Some delay also happened in posting the patient for surgery due to limited OT time.

All twenty patients completed the minimum follow up period of three months. Flynn's criteria was used to assess their clinical outcome (Table 2). 7 (35\%) patients had satisfactory results while $13(65 \%)$ patients were found to have unsatisfactory results. Among 13 patients, loss of ROM was responsible for unsatisfactory results in $10(50 \%)$ patients, loss of carrying angle in one $(5 \%)$ patient while two $(10 \%)$ patients were having unsatisfactory results both in terms of loss of ROM as well as of carrying angle.

Table 2: Flynn's criteria at three months follow up.

\begin{tabular}{|c|c|c|}
\hline $\begin{array}{c}\text { Angle } \\
\text { (Degrees) }\end{array}$ & $\begin{array}{c}\text { Loss of rom (Patients) } \\
\mathbf{N = 2 0}\end{array}$ & $\begin{array}{c}\text { Loss of carrying angle } \\
\text { (patients) } \mathbf{N = 2 0}\end{array}$ \\
\hline $0^{0}-5^{0}$ & $3(15 \%)$ & $9(45 \%)$ \\
\hline $6^{0}-10^{0}$ & $1(5 \%)$ & $5(25 \%)$ \\
\hline $11^{\mathbf{0}}-15^{\mathbf{0}}$ & $4(20 \%)$ & $3(15 \%)$ \\
\hline$>15^{0}$ & $12(60 \%)$ & $3(15 \%)$ \\
\hline
\end{tabular}

Out of the 20 patients who were included in the study, 12 patients completed the follow up period of 6 months. Flynn's criteria was again used for clinical outcome assessment in these patients (Table 3 ). We had satisfactory results in seven patients $(58.3 \%)$ while unsatisfactory results were recorded in rest of the five patients $(41.7 \%)$ as per the Flynn's criteria. four patients were having loss of ROM as the reason behind the unsatisfactory results while one patient was having unsatisfactory results due to loss of carrying angle.

Table 3: Flynn's criteria at six months follow up

\begin{tabular}{|c|c|c|}
\hline $\begin{array}{c}\text { Angle } \\
\text { (Degrees) }\end{array}$ & $\begin{array}{c}\text { Loss of rom (Patients) } \\
\mathbf{N = 1 2}\end{array}$ & $\begin{array}{c}\text { Loss of carrying angle } \\
\text { (Patients) } \mathbf{N = 1 2}\end{array}$ \\
\hline $0^{\mathbf{0}}-5^{\mathbf{0}}$ & $3(25 \%)$ & $9(75 \%)$ \\
\hline $6^{0}-10^{0}$ & $2(16.7 \%)$ & $2(16.7 \%)$ \\
\hline $11^{0}-15^{\mathbf{0}}$ & $3(25 \%)$ & 0 \\
\hline$>15^{0}$ & $4(33.3 \%)$ & $1(8.3 \%)$ \\
\hline
\end{tabular}

In the 12 patients reaching the follow up period of six months, the loss in ROM \& carrying angle at three and six months of follow up was compared. It was found that losses in range of motion as well as carrying angle decreased at six months as compared to three months. More number of patients entered in excellent and good category as per Flynn's criteria and proportion of patients having unsatisfactory range of motion decreased substantially, for which tests of statistical significance were applied. The data being skewed, Wilcoxon signed rank test was applied and the difference was found to be statistically significant ( $\mathrm{p}$ - value $<0.05$ ). 
Baumann's angle was assessed in affected as well as in normal extremity and changes were noted down. Loss of Baumann's angle was found in five patients $(25 \%)$ while 14 patients $(60 \%)$ revealed a gain in Baumann's angle, one patient had the same Baumann's angle both in normal as well as in affected extremity when measured at latest follow up. The mean Baumann's angle of immediate postop \& final follow up was compared with the mean Baumann's angle in unaffected extremity. The change was found to be statistically significant ( $p$-value $<0.05$ ). We also found that none of the patients had significant loss in reduction at latest follow up (defined by a change of more than 6 degrees between immediate post-op and latest follow-up).

Anterior humeral line was observed in all the cases and its relation in reference to capitellum was noted on a lateral radiograph of affected elbow.

The findings at immediate postoperative period and latest follow up were observed and compared. We found that AHL passed through capitellum in

$90 \%$ of the patients in the immediate post-operative period and in $85 \%$ at latest follow-up. On application of Wilcoxon signed rank test, the difference was found to be statistically insignificant ( $\mathrm{p}$-value $>0.05$ ).

The following complications were noted during our study.

Table 4: Complications during the study

\begin{tabular}{|c|c|c|}
\hline Complication & No. of patients $(\mathbf{n = 2 0})$ & \% \\
\hline Cubitus varus & 4 & 20 \\
\hline Pin site infection & 1 & 5 \\
\hline Post op serous blisters & 1 & 5 \\
\hline Neurovascular injuries & 0 & - \\
\hline Myositis ossificans & 0 & - \\
\hline Elbow stiffness & 0 & - \\
\hline malunion & 0 & - \\
\hline
\end{tabular}

Four (20\%) patients developed cubitus varus deformity while rest of the 16 patients although suffered a loss of carrying angle but elbow persisted to be in valgus position. One of our patients suffered from pin site infection which subsided after good care during pin tract dressing and oral antibiotics. One patient developed postoperative serous blisters in cubital fossa found in first check dressing on post-operative day two, which were punctured and betadine dressing was done along with oral antibiotics. Patient responded well to the treatment and they healed completely during the subsequent dressings.

None of the patients developed any sort of neurovascular injury, myositis ossificans, elbow stiffness, malunion or any other complications.

\section{Discussion}

Supracondylar humerus fractures in children have been the focus of research specially among paediatric orthopaedicians. Many studies are available over various issues associated with fracture supracondylar humerus but due to conflicting ideas and findings among authors, there is still a lack of conclusive evidence regarding the approach of management and more specifically the timing of surgery in Gartland type III Supracondylar fracture of humerus. The picture is further complicated by the fact that these fractures tend to present with a lot of swelling and associated complications for which plan may need to be individualised.

We studied 20 patients with mean age of 7.75 years with majority of the patients being males. This sort of age/sex distribution was comparable to other similar studies ${ }^{[15,16]}$ the increased male preponderance could be explained by the usual habit of boys that they do more sports activities outside home, which make them susceptible for sustaining fractures ${ }^{[17]}$.

Non dominant extremity was involved in $70 \%$ of the cases. Such epidemiological data was uniformly found in almost all studies and we found no conflict in this regard, hence our data was comparable to the previously done studies. This could be attributed to the fact that while falling down the child would have tried to cling something with the dominant hand to prevent fall. Thus, the patients fell over the non dominant extremity ${ }^{[17]}$. In our study, the mean delay in surgery was 3.9 days (SD 1.65 days, range 2-9 days) following injury. Most of the literature search reveals that authors from developed countries considers 8-12 hours as delay [2, 18-23]. While authors in developing countries have done studies with delay of days-weeks (ref of table 4 from discussion). Delay in developing countries could be explained by the fact that children suffering from any sort of fractures in developing countries most of the times tend to be ignored by the parents thinking it to be a soft tissue injury and they continue to follow home remedies till the time limb swelling increases and it becomes unbearably painful for the child. Some patents are referred to other centre, some get late due to lack of transportation facility and sometimes parents themselves are unaware about the injury.

We assessed the clinical outcome using Flynn's criteria at 3 months and 6 months follow up period. Yaokreh et al., Tahir et al. and Waikhom et al. had also used this criteria in their studies. All the three authors had better results compared to our study. The contrasting results could be attributed to the fact that our patients usually belonged to a lower socio-economic strata with most of the parents being migrant labourers. Parents didn't follow the physiotherapy routine as adviced and some parents even did local massage of the elbow after K-wire removal which leads to further loss of ROM. Moreover these authors followed up their patients for a longer period of time, which could be the reason behind their better results. Even we observed that in same group of patients at three months and six months, ROM improved significantly with compliant physiotherapy schedule.

Samal P et al. ${ }^{[16]}$ studied 58 patients with Gartland type III Supracondylar fracture of humerus who underwent closed reduction and percutaneous pinning with a mean delay in surgery of 4.5 days. The authors assessed the clinical outcome using Flynn's criteria and found that none of their study subjects were having unsatisfactory outcome which is remarkably better than our study. Closed reduction surgeries if successful do have obviously better outcomes as compared to open surgeries because it minimises soft tissue handling and periosteal damage and so helps in better healing and union at fracture site ${ }^{[24]}$

Loss in carrying angle is usually due to fixation of the fragment with a medial tilt which may not be evident in flexed elbow and is unmasked only in extension of the elbow. Loss in carrying angle could be because all patients were not operated by single surgeon and different senior residents/trainees performed the operations in emergency OT leading to heterogeneity in techniques and decision-making process. In absence of image intensifier facility in our emergency OT, at times exact assessment of reduction was not possible. Although loss of carrying angle should be comparable at 3 months and 6 months, we found differential mean values at the two instances. This finding could be attributed to the fact that some of our patients had flexion deformity at 3 months so exact carrying angle assessment was not possible in these patients. So, we got the spurious values of mean loss of carrying angle at 3 months. Therefore, we recommend that carrying angle should be 
measured after achievement of full extension at elbow joint. Pin site infection is the most frequently reported complication among almost all studies and its rate has been found to be $5-10 \%$ in these studies. Similar results were found in our study also. Even after regular pin tract dressings our patients developed pin tract infection. We recommend that pin tract dressing constitute an integral part of postoperative care and parents should be counselled regarding proper care of pin site.

During the study, we had to do open reduction in all the cases and we felt the lack of Image intensifier machine in our emergency OT. Now our centre is equipped with $\mathrm{C}$-arm machine in emergency OT which has started the practice of CRPP in our emergency OT.

Based on our unsatisfactory results in patients with delay in surgery of $>48$ hours, we conclude that these fractures should be operated as soon as the patient presents to the health care setting and a formal decision of surgery is taken. Vast literature search, supports closed reduction and percutaneous pinning as a plan of choice which can easily be done at centres equipped with $\mathrm{C}$-arm machine while open reduction and $\mathrm{K}$ - wire fixation could be a reasonably fair method in centres lacking the facility. Centres tackling these injuries should have a good protocol of physiotherapy during postoperative period. Parental counselling regarding need for physiotherapy during follow up constitutes an important part of management. Further studies need to be conducted over outcomes of early and delayed surgery in supracondylar fractures of humerus in children.

\section{References}

1. Pretell Mazzini J, Rodriguez Martin J, Andres Esteban EM. Surgical approaches for open reduction and pinning in severely displaced supracondylar humerus fractures in children: a systematic review. J Child Orthop. 2010; 4(2):143-52.

2. Carmichael KD, Joyner K. Quality of reduction versus timing of surgical intervention for pediatric supracondylar humerus fractures. Orthopedics. 2006; 29(7):628-32.

3. Mallo G, Stanat SJ, Gaffney J. Use of the Gartland classification system for treatment of pediatric supracondylar humerus fractures. Orthopedics. 2010; 33(1): 19 .

4. Gartland JJ. Management of supracondylar fractures of the humerus in children. Surg Gynecol Obstet. 1959; 109(2):145-54.

5. Loizou CL, Simillis C, Hutchinson JR. A systematic review of early versus delayed treatment for type III supracondylar humeral fractures in children. Injury. 2009; 40(3):245-8.

6. Eren A, Güven M, Erol B, Cakar M. Delayed surgical treatment of supracondylar humerus fractures in children using a medial approach. J Child Orthop. 2008; 2(1):21-7.

7. Waikhom S, Mukherjee S, Ibomcha I, Digendra A, Sohkhlet HR. Delayed Open Reduction and K-Wire Fixation of Widely Displaced Supracondylar Fractures of Humerus in Children using Medial Approach. J Clin Diagn Res. 2016; 10(8):RC06-10.

8. Tiwari A, Kanojia RK, Kapoor SK. Surgical management for late presentation of supracondylar humeral fracture in children. J Orthop Surg. 2007; 15(2):177-82.

9. Yaokreh JB, Odehouri-Koudou TH, Tembely S, Dieth AG, Kouamé DB, Ouattara $\mathrm{O}$ et al. Delayed treatment of supracondylar elbow fractures in children. Orthop Traumatol Surg Res. 2012; 98(7):808-12.

10. Naik P, Chauhan H. Delayed presentation of supracondylar humerus fractures- Open reduce now or accept for future osteotomy. Int J Paediatr Ortho. 2015; 1:23-25.

11. Flynn JC, Matthews JG, Benoit RL. Blind pinning of displaced supracondylar fractures of the humerus in children. Sixteen years' experience with long-term followup. J Bone Joint Surg Am. 1974; 56(2):263-72.

12. Camus T, MacLellan B, Cook PC. Extension type II pediatric supracondylar humerus fractures: a radiographic outcomes study of closed reduction and cast immobilization. J Pediatr Orthop. 2011; 31:366-371.

13. Shimizu T, Yoshida A, Omokawa S. Importance of anterior humeral line for successful anatomical reduction in the surgical treatment of pediatric supracondylar humeral fractures. J Orthop. 2017; 14(3):358-362.

14. Kasser JR, Beaty JH. Supracondylar fractures of the distal humerus. In: Beaty JH, Kasser JR, Wilkins KE, Rockwood CE, editors. Rockwood and Wilkins fractures in children. 6th ed. Philadelphia: Lippincott Williams and Wilkins, 2006, pp.543-89.

15. Sakthivel R, Balakrishnan V, Vadivelu G. Analysis of displaced supracondylar fractures in children treated with closed reduction and percutaneous pinning. Int $\mathrm{J}$ Res Med Sci. 2016; 4(5):1590-1596.

16. Samal P, Panigrahi T, Biswal S, Sahu MC, Mohan M, Brahma PK. Does late presentation of displaced supracondylar humerus fracture in children influence outcomes of surgery? J Clin Diagn Res. 2017; 11(10):RC09-RC13.

17. Hassan FO. Hand dominance and gender in forearm fractures in children. Strategies Trauma Limb Reconstr. 2008; 3(3):101-103.

18. Gupta N, Kay RM, Leitch K. Effect of surgical delay on perioperative complications and need for open reduction in supracondylar humerus fractures in children. J Pediatr Orthop. 2004; 24:245-8.

19. Mehlman CT, Strub WM, Roy DR. The effect of surgical timing on the perioperative complications of treatment of supracondylar humeral fractures in children. J Bone Joint Surg Am. 2001; 83-A:323-7.

20. Leet AI, Frisancho J, Ebramzadeh E. Delayed treatment of type 3 supracondylar humerus fractures in children. J Pediatr Orthop. 2002; 22:203-7.

21. Iyengar SR, Hoffinger SA, Townsend DR. Early versus delayed reduction and pinning of type III displaced supracondylar fractures of the humerus in children: a comparative study. J Orthop Trauma. 1999; 13:51-5.

22. Sibinski M, Sharma H, Bennet GC. Early versus delayed treatment of extension type-3 supracondylar fractures of the humerus in children. J Bone Joint Surg Br. 2006; 88:380-1.

23. Walmsley PJ, Kelly MB, Robb JE et al. Delay increases the need for open reduction of type-III supracondylar fractures of the humerus. J Bone Joint Surg Br. 2006; 88:528-30.

24. Ozkoc G, Gonc U, Kayaalp A, Teker K, Peker TT. Displaced supracondylar humeral fractures in children: open reduction vs. closed reduction and pinning. Arch Orthop Trauma Surg. 2004; 124(8):547-551. 